KYUNGPOOK Math. J. 48(2008), 323-330

\title{
On the Finiteness and Non-Existence of Certain Mod 2 Galois Representations of Quadratic Fields
}

\author{
Hyunsuk MOON \\ Department of Mathematics, College of Natural Sciences, Kyungpook National Uni- \\ versity, Daegu 702-701, Korea \\ e-mail : hsmoon@knu.ac.kr \\ Yuichiro TAGUCHI \\ Graduate School of Mathematics, Kyushu University 33, Fukuoka 812-8581, Japan \\ e-mail : taguchi@math.kyushu-u.ac.jp
}

ABstract. Some finiteness and non-existence results are proved of 2-dimensional mod 2 Galois representations of quadratic fields unramified outside 2.

\section{Introduction}

Let $F$ be a quadratic extension of the rational number field $\mathbb{Q}$, and $G_{F}=$ $\operatorname{Gal}(\bar{F} / F)$ the absolute Galois group of $F$. In this paper, we present some results on the finiteness and non-existence of continuous irreducible 2-dimensional mod 2 Galois representations $\rho: G_{F} \rightarrow \mathrm{GL}_{2}\left(\overline{\mathbb{F}}_{2}\right)$ unramified outside $\{2, \infty\}$ or $\{2\}$, where $\overline{\mathbb{F}}_{2}$ is an algebraic closure of the finite field $\mathbb{F}_{2}$ of two elements. This extends some of the previous results [30], [26], [3], [12], [13], [14], [15], [16], [4]. Such studies are motivated by Serre's modularity conjecture ([24], [27]) as explained in more detail below. Also, such results may be regarded as a generalization of both the HermiteMinkowski theorem and the finiteness of ideal class groups. A typical result is as follows:

Theorem 1. Let $F$ be one of the following quadratic fields:

$$
\mathbb{Q}(\sqrt{-1}), \mathbb{Q}(\sqrt{ \pm 2}), \mathbb{Q}(\sqrt{ \pm 3}), \mathbb{Q}(\sqrt{ \pm 5}), \mathbb{Q}(\sqrt{ \pm 6}) .
$$

Then there exist no continuous irreducible representations $\rho: G_{F} \rightarrow \mathrm{GL}_{2}\left(\overline{\mathbb{F}}_{2}\right)$ unramified outside $\{2, \infty\}$.

This was proved in [17], and also in [23] using the discriminant bounds in [17]. Instead of repeating its proof, we present in Section 3 the proofs of some variants of

Received February 27, 2008.

2000 Mathematics Subject Classification: 11R39, 11F80, 11R32.

Key words and phrases: Galois representation, finiteness, non-existence, discriminant bound.

This is a revised version of a manuscript included in: Diophantine analysis and related fields (DARF 2007/2008), AIP Conf. Proc. 976, Amer. Inst. Phys., Melville, NY, 2008. 
this theorem. The basic principle of the proofs is the same as in [30]; if there were a representation $\rho$ as in the theorem and $K / F$ is the extension cut out by $\rho$ (i.e. the extension corresponding to $\operatorname{Ker}(\rho)$ by Galois theory), then we compare two estimates for the root discriminant $\left|d_{K}\right|^{1 / n}$, where $n=[K: \mathbb{Q}]$, in the opposite directions, one from above and the other from below. The estimate from above, which we call the Tate bound, uses local class field theory, and is algebraic in nature. As the estimate from below, Tate [30] originally used the Minkowski bound $([29], \S 5.2)$, which is based on the geometry of numbers. Later, Serre [26] replaced this by the Odlyzko bound ([18], [21], [19]) to have better bounds; the Odlyzko bound is based on Weil's explicit formula ([31]) and highly analytic in nature. The non-existence of $\rho$ follows from the contradiction of the two inequalities (i.e. "upper bound $<$ lower bound").

The Odlyzko bound for $\left|d_{K}\right|^{1 / n}$ depends on the value of $n=[K: \mathbb{Q}]$. Sometimes, one obtains the contradiction only for sufficiently large $n$. In such a case, we can conclude the finiteness of the set of isomorphism classes of such $\rho$ 's because, by the Hermite-Minkowski theorem, for a given $n$, there exist only finitely many Galois extensions $K / \mathbb{Q}$ of degree $\leq n$ unramified outside a given finite set of places of $\mathbb{Q}$ and, for each $K / \mathbb{Q}$, there exist only finitely many irreducible representations $\rho: \operatorname{Gal}(K / \mathbb{Q}) \rightarrow \mathrm{GL}_{2}\left(\overline{\mathbb{F}}_{2}\right)$. A typical theorem in this direction is the following, in which (and elsewhere) the GRH stands for the Generalized Riemann Hypothesis for Dedekind zeta functions and "unconditionally" means "without assuming the GRH":

Theorem 2. Assume the GRH. Let $F$ be the quadratic field $\mathbb{Q}(\sqrt{M})$, where $M$ is one of

$$
\begin{aligned}
& \pm 7, \pm 10, \pm 11, \pm 13, \pm 14, \pm 15,-17, \pm 19, \\
& \quad \pm 21, \pm 22,23, \pm 26, \pm 29, \pm 30,31,-35 .
\end{aligned}
$$

Then there exist only finitely many isomorphism classes of continuous semi-simple representations $\rho: G_{F} \rightarrow \mathrm{GL}_{2}\left(\overline{\mathbb{F}}_{2}\right)$ unramified outside $\{2, \infty\}$. For $F=\mathbb{Q}(\sqrt{7})$, the finiteness holds true unconditionally.

This, together with its variants, will be proved at the end of Section 3.

Now we explain the motivation for our study in more detail. The strongest motivation for us has been Serre's conjecture ([24], [27]) on the modularity of odd irreducible mod $p$ Galois representations $\rho: G_{\mathbb{Q}} \rightarrow \mathrm{GL}_{2}\left(\overline{\mathbb{F}}_{p}\right)$ of the rational number field ("odd" means that the value of $\operatorname{det} \rho$ at a complex conjugation is -1 ). It asserts that any such $\rho$ comes from a cuspidal (elliptic modular) eigenform of level $N(\rho)$, weight $k(\rho)$ and Nebentypus character $\varepsilon(\rho)$. Here, $N(\rho)$ is the Artin conductor of $\rho$ outside $p$ (which is defined from the ramification data of $\rho$ at primes $q \neq p), k(\rho)$ is an integer with $1 \leq k(\rho) \leq p^{2}-1$ called the Serre weight (which is defined from the ramification data of $\rho$ at $p$ ), and $\varepsilon(\rho)$ is the unique character $\varepsilon(\rho):(\mathbb{Z} / N(\rho) \mathbb{Z})^{\times} \rightarrow \overline{\mathbb{F}}_{p}^{\times}$such that $\operatorname{det} \rho=\chi^{k(\rho)} \varepsilon(\rho)$ where $\chi: G_{\mathbb{Q}} \rightarrow \mathbb{F}_{p}^{\times}$is the mod $p$ cyclotomic character (for more detail on these invariants, see [27]; see also [7] for the Serre weight). Tate [30] (resp. Serre [26]; resp. Brueggeman [3] assuming the GRH) proved that there exist no irreducible representations $\rho: G_{\mathbb{Q}} \rightarrow \mathrm{GL}_{2}\left(\overline{\mathbb{F}}_{p}\right)$ 
for $p=2$ (resp. $p=3$; resp. $p=5$ ) unramified outside $\{p, \infty\}$. This proves Serre's conjecture for $N(\rho)=1$ and $p=2,3$ (and 5 under GRH). The conjecture has recently been proved completely by Khare and Wintenberger ([11]). Their proof is a kind of induction on $p, N(\rho)$ and $k(\rho)$, of which the first step is the above results of Tate and Serre.

On the other hand, various generalizations of Serre's conjecture has been proposed since before. Ash-Sinnott [2] and Ash-Doud-Pollack [1] formulated a conjecture on the modularity of higher-dimensional representations $\rho: G_{\mathbb{Q}} \rightarrow \mathrm{GL}_{n}\left(\overline{\mathbb{F}}_{p}\right)$, which associates the $\rho$ 's with Hecke eigenclasses in certain cohomology groups of congruence subgroups of $\mathrm{GL}_{n}(\mathbb{Z})$. Figueiredo asks in $[8]$ a question about the modularity of representations $\rho: G_{F} \rightarrow \mathrm{GL}_{2}\left(\overline{\mathbb{F}}_{p}\right)$ when $F$ is an imaginary quadratic field. Buzzard, Diamond and Jarvis formulated in [5] a modularity conjecture for totally real fields $F$ and odd irreducible representations $\rho: G_{F} \rightarrow \mathrm{GL}_{2}\left(\overline{\mathbb{F}}_{p}\right)$ when $p$ is unramified in $F / \mathbb{Q}$. Schein [22] extended this to the case where $p$ may be ramified in $F / \mathbb{Q}$. Our Theorem 1 implies some special cases of the conjectures of Figueiredo, Buzzard-Diamond-Jarvis and Schein.

Serre's conjecture implies in particular the finiteness of the set of isomorphism classes of odd semi-simple representations $\rho: G_{\mathbb{Q}} \rightarrow \mathrm{GL}_{2}\left(\overline{\mathbb{F}}_{p}\right)$ with $N(\rho)$ dividing a given integer $N \geq 1$, since the space of modular forms of level $N$ and weight $\leq p^{2}-1$ is finite-dimensional. Generalizing this, Khare ([10]) and one of the authors ([12]) formulated the following finiteness conjecture:

Conjecture. For any finite extension $F$ of $\mathbb{Q}$, an integer $n \geq 1$, a prime number $p$, and an integral ideal $N$ of $F$, there exist only finitely many isomorphism classes of continuous semi-simple representations $\rho: G_{F} \rightarrow \mathrm{GL}_{n}\left(\overline{\mathbb{F}}_{p}\right)$ with $N(\rho) \mid N$.

In [12], the finiteness was proved for $F=\mathbb{Q}, N(\rho)=1$ and some small values of $n$ and $p$. In [15], we proved the finiteness for those $\rho$ 's with solvable images. This proof consists in a combination of class field thoery (finiteness of the ray class groups), the Hermite-Minkowski theorem and group theory. Our Theorem 2 contributes (under GRH) to the Conjecture in some cases of quadratic fields $F$.

\section{The Tate and Odlyzko bounds}

2.1. Upper bound. Let $F=\mathbb{Q}(\sqrt{M})$ be a quadratic field, where $M$ is a square free integer. We follow Tate's method of discriminant bound ([30]). The estimate of the root discriminat $\left|d_{K}\right|^{1 / n}$ of the extension field $K$ cut out by $\rho: G_{F} \rightarrow \mathrm{GL}_{2}\left(\overline{\mathbb{F}}_{2}\right)$ reduces to those of local extensions $K_{\mathfrak{P}} / F_{\mathfrak{p}}$, where $\mathfrak{p}$ (resp. $\mathfrak{P}$ ) is a prime of $F$ (resp. $K$ ) lying above 2 (resp. $\mathfrak{p}$ ). If $K_{\mathfrak{P}} / F_{\mathfrak{p}}$ is tamely ramified, then its different has 2 -adic order $<1$. So we only need to consider the wildly ramified cases. The local Lemmas 2 and 3 of [17] and Proposition 2.3 of [20] imply the following (use these lemmas and proposition respectively for the cases (i), (ii) and (iii) below): 
Lemma 1. Assume $K_{\mathfrak{P}} / F_{\mathfrak{p}}$ has wild ramification index $\leq 2^{m}$ at all $\mathfrak{p} \mid 2$.

(i) If $F / \mathbb{Q}$ is ramified at 2 , then

$$
\left|d_{K}\right|^{1 / n} \leq \begin{cases}2^{11 / 4} \sqrt{|M|} & \text { if } m \leq 2, \\ 2^{23 / 8} \sqrt{|M|} & \text { if } m \leq 3, \\ 8 \sqrt{|M|} & \text { in general }\end{cases}
$$

(ii) If $F / \mathbb{Q}$ is inert at 2 , then

$$
\left|d_{K}\right|^{1 / n} \leq \begin{cases}2^{8 / 3} \sqrt{|M|} & \text { if } m \leq 2, \\ 2^{35 / 12} \sqrt{|M|} & \text { in general } .\end{cases}
$$

(iii) If $F / \mathbb{Q}$ is split at 2 , then

$$
\left|d_{K}\right|^{1 / n} \leq 16 \sqrt{|M|} \quad \text { in general. }
$$

2.2. Lower bound. Let $K$ be an algebraic number field of finite degree $n=$ $[K: \mathbb{Q}]$. For some relevant values of $n$, we simply extract the following data from Odlyzko's table [19]:

Lemma 2. (i) Unconditionally, we have, for a general $K$,

$$
\left|d_{K}\right|^{1 / n}> \begin{cases}17.020 & \text { if } n \geq 120 \\ 20.895 & \text { if } n \geq 1000, \\ 21.845 & \text { if } n \geq 4840\end{cases}
$$

and, for a totally real $K$,

$$
\left|d_{K}\right|^{1 / n}> \begin{cases}43.513 & \text { if } n \geq 120 \\ 55.966 & \text { if } n \geq 1000 \\ 59.079 & \text { if } n \geq 4840 \\ 60.702 & \text { if } n \geq 10^{7}\end{cases}
$$

(ii) Assuming the GRH, we have, for a general K,

$$
\left|d_{K}\right|^{1 / n}> \begin{cases}20.221 & \text { if } n \geq 120, \\ 29.094 & \text { if } n \geq 1000,\end{cases}
$$

and, for a totally real $K$,

$$
\left|d_{K}\right|^{1 / n}> \begin{cases}63.335 & \text { if } n \geq 120 \\ 107.548 & \text { if } n \geq 1000\end{cases}
$$


Serre gave the following better asymptotic bound (as $n \rightarrow \infty$ ) under the GRH $([25])$ :

Lemma 3. Assuming the GRH, we have:

$$
\left|d_{K}\right|^{1 / n}> \begin{cases}44.7 & \text { for a general } K, \\ 215.3 & \text { for a totally real } K,\end{cases}
$$

if $n$ is sufficiently large.

\section{The results}

First we give some results which supplement Theorem 1.

Theorem 3. Assume the GRH. If $F=\mathbb{Q}(\sqrt{7})$, there exists no irreducible representation $\rho: G_{F} \rightarrow \mathrm{GL}_{2}\left(\overline{\mathbb{F}}_{2}\right)$ unramified outside $\{2, \infty\}$. Unconditionally, if such a $\rho$ exists, then $\operatorname{Im}(\rho)$ is isomorphic to $\mathrm{SL}_{2}\left(\mathbb{F}_{4}\right)$.

Proof. The case where $\operatorname{Im}(\rho)$ is solvable can be handled in the same way as in [17]. If $G=\operatorname{Im}(\rho)$ is solvable, then it sits in an exact sequence

$$
1 \rightarrow H \rightarrow G \rightarrow \mathbb{Z} / 2 \mathbb{Z} \rightarrow 1, \quad H \subset \overline{\mathbb{F}}_{2}^{\times} \times \overline{\mathbb{F}}_{2}^{\times},
$$

as in Theorem 1 in $\S 22$ of [28]. Hence $K$ is an abelian extension of odd degree, unramified outside $\{2, \infty\}$, over the quadratic extension $K^{\prime} / F$ corresponding to $H$. By using class field theory, there are 3 possibilities for such $K^{\prime}$. By examining Jones' tables [9], we find them as follows:

$$
K^{\prime}=\mathbb{Q}(\sqrt{7}, \sqrt{-1}), \mathbb{Q}(\sqrt{7}, \sqrt{2}), \mathbb{Q}(\sqrt{7}, \sqrt{-2}) .
$$

All these $K^{\prime}$ have class number 1 . Since any ray class group of $K^{\prime}$ of 2-power conductor has 2-power order, there is no non-trivial abelian extension $K / K^{\prime}$ of odd degree unramified outside $\{2, \infty\}$.

Next we prove the non-solvable case. If $\operatorname{Im}(\rho)$ is non-solvable, then $|\operatorname{Im}(\rho)| \geq 60$, and hence $n=[K: \mathbb{Q}] \geq 120$. Further, $|\operatorname{Im}(\rho)|$ can be bounded from below as follow: By $\S \S 251-253$ of [6], if the 2-Sylow subgroup of $\operatorname{Im}(\rho)$ has order $2^{\mu}$, then its image in $\mathrm{PGL}_{2}\left(\overline{\mathbb{F}}_{2}\right) \simeq \mathrm{SL}_{2}\left(\overline{\mathbb{F}}_{2}\right)$ coincides with a conjugate of $\mathrm{SL}_{2}\left(\mathbb{F}_{2^{\mu}}\right)$. Thus if $K / F$ has wild ramification index $2^{m}$ at a prime above 2 , then $|\operatorname{Im}(\rho)| \geq\left|\mathrm{SL}_{2}\left(\mathbb{F}_{2^{m}}\right)\right|$. Now, since $\mathbb{Q}(\sqrt{7}) / \mathbb{Q}$ is ramified at 2 , we obtain a contradiction by comparing (i) of Lemma 1 and $(3)$ of Lemma 2.

Unconditionally, we obtain a contradiction except the case $m \leq 2$ and $\operatorname{Im}(\rho)$ does not contain a conjugate of $\mathrm{SL}_{2}\left(\mathbb{F}_{8}\right)$. In this case, $\operatorname{Im}(\rho)$ contains $\mathrm{SL}_{2}\left(\mathbb{F}_{4}\right)$, and its image in $\mathrm{PGL}_{2}\left(\overline{\mathbb{F}}_{2}\right)$ is isomorphic to $\mathrm{SL}_{2}\left(\mathbb{F}_{4}\right)$. But we have $\operatorname{det} \rho=1$, since there is no character $G_{F} \rightarrow \overline{\mathbb{F}}_{2}^{\times}$unramified outside $\{2, \infty\}$, as can be seen from the fact that $\left(\mathcal{O}_{F} \otimes_{\mathbb{Z}} \mathbb{Z}_{2}\right)^{\times}$is a pro-2 group by using class field theory (Here, $\mathcal{O}_{F}$ is the integer ring of $F$ ). Thus $\operatorname{Im}(\rho)$ itself must be isomorphic to $\mathrm{SL}_{2}\left(\mathbb{F}_{4}\right)$. 
Similarly, we have:

Theorem 4. Assume the GRH.

(1) Let $F=\mathbb{Q}(\sqrt{-11})$. If there is a representation $\bar{\rho}: G_{F} \rightarrow \mathrm{PGL}_{2}\left(\overline{\mathbb{F}}_{2}\right)$ unramified outside $\{2, \infty\}$, then $\operatorname{Im}(\bar{\rho})$ is isomorphic to $\mathrm{SL}_{2}\left(\mathbb{F}_{4}\right)$.

(2) Let $F=\mathbb{Q}(\sqrt{13})$. If there is a representation $\bar{\rho}: G_{F} \rightarrow \mathrm{PGL}_{2}\left(\overline{\mathbb{F}}_{2}\right)$ unramified outside $\{2, \infty\}$ and with non-solvable image, then $\operatorname{Im}(\bar{\rho})$ is isomorphic to $\mathrm{SL}_{2}\left(\mathbb{F}_{4}\right)$.

Since the proof is basically the same as that of Theorem 3, we only note here some relavant facts in the proof. In the non-solvable case, we obtain a contradiction if $n \geq 2 \times\left|\mathrm{SL}_{2}\left(\mathbb{F}_{8}\right)\right|$, by comparing (ii) of Lemma 1 and (3) of Lemma 2 .

In the case of $F=\mathbb{Q}(\sqrt{-11})$ and $\operatorname{Im}(\rho)$ is solvable, the possible quadratic extensions $K^{\prime}$ of $F$ corresponding to $H$ of $(*)$ are $K^{\prime}=\mathbb{Q}(\sqrt{-11}, \sqrt{-1}), \mathbb{Q}(\sqrt{-11}, \sqrt{2})$, $\mathbb{Q}(\sqrt{-11}, \sqrt{-2})$. They all have class number 1 . In the case of $F=\mathbb{Q}(\sqrt{13})$, there are 7 possibilities for the quadratic extension $K^{\prime}$ corresponding to $H$ : $K^{\prime}=$ $\mathbb{Q}(\sqrt{(-3 \pm \sqrt{13}) / 2}), \mathbb{Q}(\sqrt{-3 \pm \sqrt{13}}), \mathbb{Q}(\sqrt{13}, \sqrt{-1}), \mathbb{Q}(\sqrt{13}, \sqrt{2}), \mathbb{Q}(\sqrt{13}, \sqrt{-2})$. The field $K^{\prime}=\mathbb{Q}(\sqrt{13}, \sqrt{-2})$ has class number 3, and the other six fields have class number 1 .

Next we consider representations $\rho$ of $G_{F}$ unramified also at $\infty$ for real quadratic fields $F$. In this case, the extensions $K / F$ cut out by $\rho$ are totally real, and hence we have larger values $\left((2)\right.$ and (4) of Lemma 2) as the lower bounds of $\left|d_{K}\right|^{1 / n}$.

Theorem 5. (i) (Unconditionally) Let $F$ be the real quadratic field $\mathbb{Q}(\sqrt{M})$ with one of the following values of $M$ :

$$
7,10,11,13,14,15,19,21,22,23,26,29,30,31,34,35,37,38,39 .
$$

Then there exist no representations $\rho: G_{F} \rightarrow \mathrm{GL}_{2}\left(\overline{\mathbb{F}}_{2}\right)$ unramified outside $\{2\}$ with non-solvable images.

(ii) Assume the GRH. Let $F$ be a real quadratic field $\mathbb{Q}(\sqrt{M})$ such that either $(M \leq 87$ and $F / \mathbb{Q}$ is ramified at 2$)$ or $(M \leq 93$ and $F / \mathbb{Q}$ is inert at 2$)$. Then there exist no representations $\rho: G_{F} \rightarrow \mathrm{GL}_{2}\left(\overline{\bar{F}}_{2}\right)$ unramified outside $\{2\}$ with nonsolvable images.

Proof. Compare (i), (ii) of Lemma 1and (2), (4) of Lemma 2.

Finally, we trun to the finiteness. Theorem 2 can be proved by comparing Lemma 1 and Lemma 3. Similarly, we can prove:

Theorem 6. (i) (Unconditionally) Let $F$ be a real quadratic field $\mathbb{Q}(\sqrt{M})$ such that either $(M \leq 55$ and $F / \mathbb{Q}$ is ramified at 2$)$ or $(M \leq 61$ and $F / \mathbb{Q}$ is inert at 2$)$. Then there exist only finitely many isomorphism classes of semi-simple representations $\rho: G_{F} \rightarrow \mathrm{GL}_{2}\left(\overline{\mathbb{F}}_{2}\right)$ unramified outside $\{2\}$.

(ii) Assume the GRH. Let $F$ be a real quadratic field $\mathbb{Q}(\sqrt{M})$ such that either $(M \leq 723$ and $F / \mathbb{Q}$ is ramified at 2$),(M \leq 805$ and $F / \mathbb{Q}$ is inert at 2$)$, or $(M \leq 161$ and $F / \mathbb{Q}$ is split at 2$)$. Then there exist only finitely many isomorphism 
classes of semi-simple representations $\rho: G_{F} \rightarrow \mathrm{GL}_{2}\left(\overline{\mathbb{F}}_{2}\right)$ unramified outside $\{2\}$.

Acknowledgment. The second author was supported by JSPS Grant 19540036 and JSPS Core-to-Core Program (Coordinator: Makoto Matsumoto) while working on the subject of this paper.

\section{References}

[1] A. Ash, D. Doud and D. Pollack, Galois representations with conjectural connections to arithmetic cohomology, Duke Math. J., 112(2002), 521-579.

[2] A. Ash and W. Sinnott, An analogue of Serre's conjecture for Galois representations and Hecke eigenclasses in the mod $p$ cohomology of $\mathrm{GL}(n, Z)$, Duke Math. J., 105(2000), 1-24.

[3] S. Brueggeman, The nonexistence of certain Galois extensions unramified outside 5, J. Number Theory, 75(1999), 47-52.

[4] S. Brueggeman, The nonexistence of certain nonsolvable Galois extensions of number fields of small degree, Int. J. Number Theory, 1(2005), 155-160.

[5] K. Buzzard, F. Diamond and F. Jarvis, On Serre's conjecrure for mod $\ell$ Galois representations over totally real fields, preprint, 2005, available at: http://www . unet. brandeis. edu/ ${ }^{\sim}$ fdiamond/bdj12.pdf

[6] L. E. Dickson, Linear Groups, Teubner, 1901, Leibzig.

[7] B. Edixhoven, The weight in Serre's conjectures on modular forms, Invent. Math., 109(1992), 568-594.

[8] L. M. Figueiredo, Serre's conjecture for imaginary quadratic fields, Compositio Math., 118(1999), 103-122.

[9] J. Jones, Tables of number fields with prescribed ramification, http://math. asu.edu/ jj/numberfields/

[10] C. Khare, Conjectures on finiteness of mod $p$ Galois representations, J. Ramanujan Math. Soc., 15(2000), 23-42.

[11] C. Khare and J.-P. Wintenberger, Serre's modularity conjecture, I and II, preprint, available at: http://www.math.utah.edu/ shekhar/papers.html

[12] H. Moon, Finiteness results on certain mod $p$ Galois representations, J. Number Theory, 84(2000), 156-165.

[13] H. Moon, The non-existence of certain mod $p$ Galois representations, Bull. Korean Math. Soc., 40(2003), 537-544.

[14] H. Moon, On mod 3 Galois representations with conductor 4, Proc. Amer. Math. Soc., 135(2007), 3109-3113.

[15] H. Moon and Y. Taguchi, Mod p Galois representations of solvable image, Proc. Amer. Math. Soc., 129(2001), 2529-2534. 
[16] H. Moon and Y. Taguchi, Refinement of Tate's discriminant bound and non-existence theorems for mod $p$ Galois representations, Kazuya Kato's fiftieth birthday, Doc. Math. Extra Vol. (2003), 641-654.

[17] H. Moon and Y. Taguchi, The non-existence of certain mod 2 Galois representations of some small quadratic fields, Proc. Japan Acad. 84, Ser. A(2008), 57-61.

[18] A. Odlyzko, Lower bounds for discriminants of number fields. II, Tôhoku Math. J., 29(1977), 209-216.

[19] A. M. Odlyzko, Discriminant bounds, unpublished manuscript, 1976, available at: http://www.dtc.umn.edu/ odlyzko/unpublished/index.html

[20] K. Ono and Y. Taguchi, 2-adic properties of certain modular forms and their applications to arithmetic functions, Int. J. Number Theory, 1(2005), 75-101.

[21] G. Poitou, Minorations de discriminants (d'après A. M. Odlyzko), Sém. Bourbaki, 1975/76, Exp. 479, pp. 136-153, Lecture Notes in Math. 567, Springer, Berlin, 1977.

[22] M. Schein, Weights in Serre's conjecture for Hilbert modular forms: the ramified case, preprint, 2006, available at: http://arxiv.org/abs/math.NT/0610488

[23] M. H. Şengün, The non-existence of certain representations of the absolute Galois group of quadratic fields, preprint, 2007.

[24] J.-P. Serre, Valeurs propres des opérateurs de Hecke modulo $\ell$, Journées Arithmétiques de Bordeaux (Conf., Univ. Bordeaux, 1974), pp. 109-117, Astérisque 24-25, Soc. Math. France, Paris, 1975.

[25] J.-P. Serre, Minorations de discriminants, Cuvres III, pp. 240-243, Springer-Verlag, 1986.

[26] J.-P. Serre, Note 229.2, CEuvres III, p. 710, Springer-Verlag, 1986.

[27] J.-P. Serre, Sur les représentations modulaires de degré 2 de Gal $(\overline{\mathbb{Q}} / \mathbb{Q})$, Duke Math. J., 54(1987), 179-230.

[28] D. A. Suprunenko, Matrix Groups, Amer. Math. Soc., Providence, 1976.

[29] T. Takagi, Algebraic Number Theory, 2nd ed. (in Japanese), Iwanami Shoten, Tokyo, 1971.

[30] J. Tate, The non-existence of certain Galois extensions of $\mathbb{Q}$ unramified outside 2, in: "Arithmetic Geometry", pp. 153-156, Contemp. Math., 174, Amer. Math. Soc., Providence, RI, 1994.

[31] A. Weil, Sur les "formules explicites" de la théorie des nombres premiers, Comm. Sém. Math. Univ. Lund, Tome Supplementaire (1952), 252-265. 